\title{
Prévalence de la fasciolose bovine dans la vallée du fleuve Niger au Bénin
}

\author{
A.K.I. Youssao ${ }^{1 *}$ M.N. Assogba ${ }^{2}$
}

\section{Mots-clés}

Bovin - Fasciola gigantica Morbidité - Fleuve Niger - Bénin.

\begin{abstract}
Résumé
Une enquête sur la fasciolose bovine a été réalisée en octobre 1995 et en mars 1996 dans la vallée du fleuve Niger au Bénin en vue d'évaluer l'importance de cette maladie. Pour l'analyse coprologique, la méthode qualitative avec concentration par sédimentation simple a été utilisée. L'aspect général du foie a d'abord été examiné et le parenchyme hépatique a ensuite été incisé pour extraire les grandes douves logées dans les canaux biliaires. Les résultats des analyses coprologiques ont indiqué des prévalences qui ont varié entre 7,5 à 52,4 p. 100 en fonction des localités et du mois de l'année. Aux abattoirs de Malanville, la prévalence des foies parasités par Fasciola gigantica a été d'environ 30 p. 100 quelle qu'ait été la saison.
\end{abstract}

\section{INTRODUCTION}

Long de $4200 \mathrm{~km}$, le fleuve Niger est le troisième grand fleuve d'Afrique après le Nil et le Congo, et le plus important en Afrique occidentale. Des enquêtes malacologiques réalisées le long de ce fleuve (Niger moyen) et dans les mares de sa vallée ont permis de mettre en évidence des gastéropodes appartenant aux genres Lymnaea, Biomphalaria et Bulinus (5). Lymnaea natalensis est l'hôte intermédiaire de Fasciola gigantica, agent causal de la fasciolose en Afrique de l'Ouest. D'un point de vue sanitaire et économique, la fasciolose est une maladie considérée comme la parasitose la plus dangereuse des ruminants domestiques en Afrique. Au Bénin, la fasciolose à Fasciola gigantica est endémique dans le bassin du fleuve Niger et plusieurs cas ont été observés dans la région (1, 2, 7). Cette étude a été réalisée pour connaître davantage l'importance de cette parasitose dans les troupeaux et dans les abattoirs de la vallée du Niger moyen.

\footnotetext{
1. Faculté de médecine vétérinaire, Institut vétérinaire tropical, $20 \mathrm{Bd}$ de Colonster, B 43, 4000 Liège, Belgique

2. Université d'Abomey-Calavi, Collège polytechnique universitaire, département des Productions animales, BP 2009, Cotonou, Bénin

* Auteur pour la correspondance

Faculté de médecine vétérinaire, service de Biostatistique (B43),

$20 \mathrm{Bd}$ de Colonster, 4000 Liège, Belgique

Tél. : +32(0)43664091 ou +32(0)43664150; fax : +32(0)4 3664122

E-mail : iyoussao@student.ulg.ac.be
}

\section{MATERIEL ET METHODES}

\section{Milieu de l'étude}

Le fleuve Niger est situé dans l'extrême nord du Bénin et représente la frontière entre le Bénin et le Niger (figure 1). Son débit est de $1058 \mathrm{~m}^{2} / \mathrm{s}$ dans le Niger moyen, à Malanville. Le climat est de type soudano-sahélien avec une saison des pluies (900 mm par an) de mai à septembre et une saison sèche de novembre à avril. Au moment du retrait des eaux en début de saison sèche, de vastes superficies se couvrent de végétations (bourgoutières) particulièrement appréciées par les ruminants. Les pâturages des vallées du Niger sont utilisés pendant la sécheresse. Les zébus sont les bovins les plus rencontrés et la transhumance est la pratique la plus répandue. L'embouche bovine est également pratiquée dans cette région.

\section{Collecte et analyse des prélèvements coprologiques}

Des prélèvements de fèces ont été réalisés sur 236 bovins en octobre 1995, à la fin de la saison des pluies, et sur 220 en mars 1996, en pleine saison sèche. Les troupeaux des villes de Karimama et Malanville, situés aux abords ou aux environs du fleuve Niger, ont été choisis pour les prélèvements. Il en a été de même pour les troupeaux de Gaya, situés à six kilomètres de Malanville dans le territoire nigérien et en bordure du fleuve Niger.

Le prélèvement des matières fécales a été réalisé par voie rectale entre 7 et $9 \mathrm{~h}$, avant la conduite des animaux au pâturage. Dès qu'une quantité suffisante de matières fécales a été prélevée, 


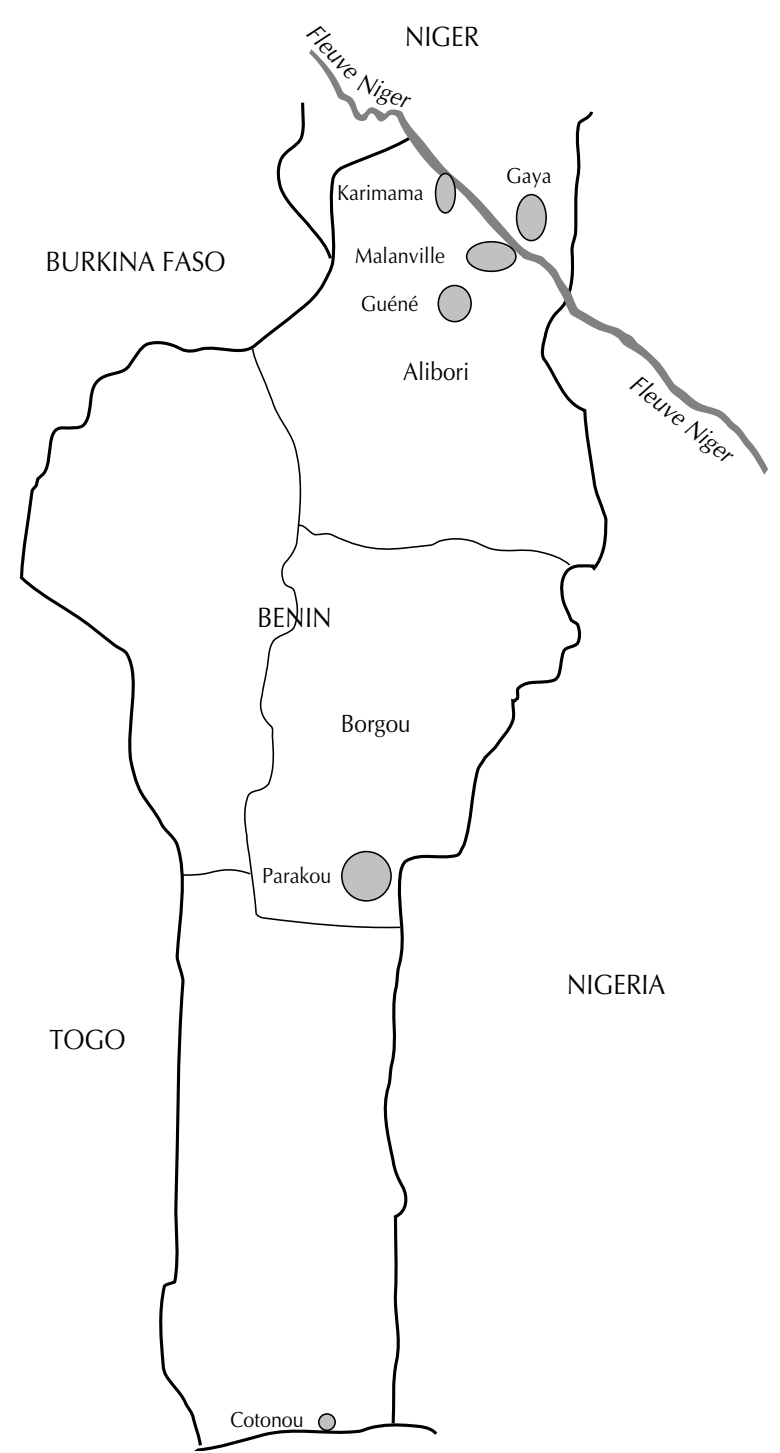

Figure 1 : situation géographique du fleuve Niger au Bénin.

le sachet a été retourné et soigneusement noué. La conservation des prélèvements a été réalisée à $+4{ }^{\circ} \mathrm{C}$ pendant quelques jours avant l'observation des œufs des parasites au laboratoire. La technique utilisée pour l'analyse microscopique a été la méthode qualitative avec concentration par sédimentation simple (6). Cette technique a per-

\section{Examen des foies}

L'examen de foies a eu lieu à l'abattoir de Malanville (Bénin). Les animaux abattus étaient originaires des sous-préfectures de Karimama et Malanville (Bénin) et de la région de Gaya (Niger). En moyenne, 12 bovins ont été abattus par jour. Après l'examen de l'aspect général du foie, de l'hypertrophie et de l'angiocholite, le parenchyme hépatique a été incisé. Une pression manuelle a été exercée sur le parenchyme pour extraire les grandes douves (Fasciola gigantica) logées dans les canaux biliaires. La période de dissection, l'espèce animale, le nombre de foies examinés et le nombre de foies infestés ont été notés. Parmi les foies infestés, ceux qui contenaient moins de 20 grandes douves ont été classés dans le groupe des animaux les moins infestés. Ceux qui étaient moyennement infestés contenaient 20 à 50 grandes douves. Enfin, les bovins dont le foie contenaient plus de 50 grandes douves ont été classés dans le groupe de ceux qui étaient fortement infestés.

\section{Analyses statistiques}

La prévalence est le pourcentage du nombre d'animaux parasités sur l'effectif total des animaux. Pour l'évaluer, les facteurs de variation retenus ont été, d'une part, le mois de prélèvement des matières fécales pour la coprologie (octobre 1995 et mars 1996) et, d'autre part, le mois d'abattage (octobre 1995 et mars 1996) et l'espèce animale (taurin et zébu) pour les examens de foies à l'abattoir.

Pour la coprologie, les prélèvements ont été réalisés uniquement sur des zébus. Lors de cette étude, la distribution par sexe et par âge n'a pas été abordée. Le prélèvement des matières fécales a été réalisé sur des animaux adultes en âge de reproduction (trois ans et plus). L'examen de foies a été réalisé sur les zébus et les taurins de race Borgou. L'interaction entre le mois d'abattage et la race n'a pas été significative et, par conséquent, n'a pas été prise en compte. Les données ont été analysées selon la procédure des modèles linéaires généralisés de SAS (3), puis les prévalences ont été estimées par la méthode des moindres carrés et comparées par le test de t.

\section{RESULTATS ET DISCUSSION}

Les prévalences de la fasciolose bovine dans la vallée du fleuve Niger par la méthode de coprologie sont indiquées dans le tableau I. Les prévalences obtenues en mars 1996 ont diminué significativement $(\mathrm{p}<0,001)$ dans les différentes localités par rapport à

\section{Tableau I}

Prévalence de la fasciolose bovine par la coprologie dans la vallée du fleuve Niger

\begin{tabular}{lcccccc} 
& \multicolumn{3}{c}{ Octobre 1995 } & & \multicolumn{2}{c}{ Mars 1996} \\
\hline Localité & $\begin{array}{l}\text { Effectif } \\
\text { prélevé }\end{array}$ & $\begin{array}{c}\text { Nombre de } \\
\text { cas positifs }\end{array}$ & $\begin{array}{c}\text { \% de } \\
\text { cas positifs }\end{array}$ & $\begin{array}{c}\text { Effectif } \\
\text { prélevé }\end{array}$ & $\begin{array}{c}\text { Nombre de } \\
\text { cas positifs }\end{array}$ & $\begin{array}{c}\% \text { de } \\
\text { cas positifs }\end{array}$ \\
\hline Malanville & 84 & 28 & $33,3^{\mathrm{a}}$ & 80 & 6 & $7,5^{\mathrm{b}}$ \\
Karimama & 80 & 20 & $25^{\mathrm{a}}$ & 60 & 6 & $10^{\mathrm{b}}$ \\
Gaya & 72 & 38 & $51,38^{\mathrm{a}}$ & 80 & 12 & $15^{\mathrm{b}}$ \\
Total & 236 & 86 & $36,44^{\mathrm{a}}$ & 220 & 24 & $10,91^{\mathrm{b}}$
\end{tabular}

Les valeurs sur une même ligne suivies de lettres différentes sont significativement différentes au seuil de 1 p. 100 


\section{Tableau II}

Prévalence de la fasciolose bovine à l'abattoir de Malanville dans la vallée du fleuve Niger

\begin{tabular}{lccccccc} 
& $\begin{array}{c}\text { Nb. de foies } \\
\text { examinés }\end{array}$ & $\begin{array}{c}\text { Foies infestés } \\
(\%)\end{array}$ & $\begin{array}{c}\text { Bos taurus } \\
\text { infestés (\%) }\end{array}$ & $\begin{array}{c}\text { Bos indicus } \\
\text { infestés (\%) }\end{array}$ & $\begin{array}{c}\text { Foies } \\
\text { faiblement } \\
\text { infestés (\%) }\end{array}$ & $\begin{array}{c}\text { Foies } \\
\text { moyennement } \\
\text { infestés }(\%)\end{array}$ & $\begin{array}{c}\text { Foies } \\
\text { fortement } \\
\text { infestés (\%) }\end{array}$ \\
\hline Octobre 1995 & 145 & 30,3 & $1,4^{\text {a }}$ & $28,9^{b}$ & 15,2 & 13,8 & 1,4 \\
Mars 1996 & 146 & 30,1 & $4,8^{\text {a }}$ & $25,3^{b}$ & 8,9 & 10,9 & 10,3
\end{tabular}

Les valeurs sur une même ligne suivies de lettres différentes sont significativement différentes au seuil de 1 p. 100

celles relevées en octobre 1995. La prévalence d'œufs est importante vers la fin de la saison des pluies ou au début de la saison sèche (octobre-novembre). La régression de la prévalence observée en mars pourrait être due à la disparition des formes adultes de Fasciola gigantica dans les canaux biliaires. Les résultats de cette étude confirment les observations faites par Schillhorn Van Veen (4). Cet auteur rapporte que la prévalence maximale dans un troupeau infesté par les métacercaires s'observe au début de la saison des pluies et que la maladie est communément présente à la fin de la saison des pluies jusqu'au milieu de la saison sèche. Lors de l'étude épidémiologique des principales trématodoses des animaux domestiques dans la région du fleuve Niger, Tager-Kangan (5) rapporte que dans les criques où il existe une végétation de $\mathrm{Nym}$ phea et de Sphenoclea, les limnées apparaissent fin janvier, début février (saison sèche) sur le fleuve Niger. Ces populations de limnées restent peu importantes ; elles atteignent leur maximum en mars-avril (saison sèche), puis elles décroissent rapidement au moment où le retrait des eaux intervient sur la végétation. Les bovins pâturant en janvier le long du fleuve Niger évacuent les œufs de douve, entraînant une infestation massive des bovins par les métacercaires en mars-avril. Des résultats comparables ont été rapportés par Assogba et Youssao (1).

Les prévalences de la fasciolose bovine à l'abattoir de Malanville sont indiquées dans le tableau II par espèce bovine et par degré d'infestation. En octobre 1995, sur 145 foies examinés, 30,3 p. 100 ont été infestés par $F$. gigantica. Les taurins ont été moins infestés que les zébus $(\mathrm{p}<0,001)$ quel qu'ait été le mois d'abattage. Le tableau II montre que les valeurs obtenues en octobre 1995 sur 145 foies et en mars 1996 sur 146 foies ont été semblables. La seule différence significative a résidé dans la prévalence de foies fortement infestés en mars 1996 (saison sèche) comparée à la prévalence relevée en octobre 1995 (fin de la saison des pluies). Cette différence est liée à l'élimination par les pasteurs des animaux malades, fatigués et épuisés par les déplacements, la soif et la malnutrition pendant la saison sèche.

Il ressort des résultats de cette étude que les zébus ont été plus infestés que les taurins. Cette différence a été essentiellement due à leur répartition géographique ainsi qu'à l'effectif peu élevé des taurins abattus. La quasi-totalité (90 p. 100) des bovins a été représentée par des zébus à Malanville et ses environs. Ces résultats sont comparables à ceux observés par Assogba et Youssao (2). Les résultats de la coprologie ont été similaires à ceux de l'abattoir pour le mois d'octobre 1995. En revanche, un écart très important a été observé en mars 1996 avec une prévalence trois fois plus élevée à l'abattoir de Malanville (tableaux I et II). Cette différence a été également liée à l'élimination par les pasteurs des animaux malades, fatigués et épuisés par les déplacements, la soif et la malnutrition vers la fin de la saison sèche (mars et avril).

\section{CONCLUSION}

La fasciolose bovine a été une parasitose très fréquente dans les troupeaux qui ont fréquenté la vallée du fleuve Niger. L'excrétion d'œufs de $F$. gigantica a été plus importante à la fin de la saison des pluies qu'en saison sèche. Aucune différence significative n'a été observée entre les taux d'infestation des foies en fin de saison des pluies (octobre) et en saison sèche (mars). Etant donné l'importance sanitaire et économique de cette maladie, des mesures communes doivent être prises de la source du fleuve au Fouta Dajallon en Guinée jusqu'à son embouchure au Cameroun.

\section{Remerciements}

Les auteurs remercient le professeur Pascal Leroy et les deux lecteurs anonymes pour leur contribution.

\section{BIBLIOGRAPHIE}

1. ASSOGBA M.N., YOUSSAO A.K.I., 2001. Epidémiologie de la fasciolose à Fasciola gigantica (Cobbold, 1885), de la dicrocoeliose et de la paramphistomose bovines au Bénin. Ann. Méd. vét., 145 : 260-268.

2. ASSOGBA M.N., YOUSSAO A.K.I., 2001. Prévalence de la fasciolose bovine à Fasciola gigantica (Cobbold, 1885) dans les principaux abattoirs du Bénin. Revue Méd. vét., 152 : 699-704.

3. SAS/STAT, 1989. User's guide. Vers. 6, 4th edn. Cary, NC, USA, SAS Inst.

4. SCHILLHORN VAN VEEN T.W., 1980. Fasciolosis ( $F$. gigantica) in West Africa. Rev. vet. Bull., 5: 229-233.

5. TAGER-KAGAN P., 1977. Contribution à l'étude de l'épidémiologie des principales trématodoses des animaux domestiques dans la région du fleuve Niger. Revue Elev. Méd. vét. Pays trop., 30 : 11-18.

6. THIENPONT D., ROCHETTE F., VANPARIJS O.F.J., 1995. Diagnostic de verminose par examen coprologique, $2^{\mathrm{e}}$ édn. Beerse, Belgique, Janssen Research Foundation, 205 p.

7. VISSOH K., 1980. Contribution à l'étude épizooétiologique descriptive de la fasciolose bovine en Afrique de l'Ouest : cas du nord de la République populaire du Bénin. Thèse Doct. vét., EISMV, Dakar, Sénégal, 180 p.

Reçu le 22.04.2002, accepté le 23.06.2003 


\section{Summary}

Youssao A.K.I., Assogba M.N. Prevalence of Cattle Fasciolosis in the Niger River Valley of Benin

A survey was conducted on cattle fasciolosis in the Niger River valley of Benin in October 1995 and March 1996 to assess the importance of the disease. Feces examinations were performed with the qualitative method by simple sedimentation. The overall aspect of the livers was first examined, then the hepatic parenchyma was incised to expel the large flukes that were present in the bile ducts. The results of the feces examinations showed prevalences that varied from 7.5 to $52.4 \%$ depending on the locality and the month of the year. In Malanville slaughterhouses, the prevalence of Fasciola gigantica-infested livers was about $30 \%$ regardless of the season.

Key words: Cattle - Fasciola gigantica - Morbidity - Niger River - Benin.

\section{Resumen}

Youssao A.K.I., Assogba M.N. Prevalencia de la fasciolosis bovina en el valle del río Níger, en Benin

Se llevó a cabo una encuesta sobre la fasciolosis bovina, en octubre de 1995 y en -marzo de 1996 en el valle del río Níger en Benin, esto con el fin de evaluar la importancia de esta enfermedad. Para el análisis coprológico se utilizó el método cualitativo, con concentración por sedimentación simple. El aspecto general del hígado fue primero examinado y luego se hizo una incisión en el parénquima hepático para extraer las grandes duelas de los canales biliares. Los resultados de los análisis coprológicos presentaron prevalencias que variaron entre 7,5 y $52,4 \%$, en función de las localidades y del mes del año. En los mataderos de Malanville, la prevalencia de los hígados parasitados por Fasciola gigantica fue de alrededor $30 \%$ durante cualquier estación.

Palabras clave: Ganado bovino - Fasciola gigantica - Morbosidad - Río Níger - Benin. 